\title{
Acknowledgement of top manuscript reviewers 2014
}

Antonin Bukovsky

\section{Contributing reviewers}

The editor of Reproductive Biology and Endocrinology would like to thank all of our reviewers and particularly our top reviewers who have contributed to the journal in Volume 12 (2014).

Victor Absalon-Medina

United States of America

Pilar Alamá Faubel

Spain

Erika Alvarenga

Brazil

Joao Araujo

Portugal

Silvana Arrighi

Italy

Kenneth Aston

United States of America

Graham Barrell

New Zealand

Paul Barriere

France

Cordula Bartel

Austria

Natalia Basile

Spain

Yaakov Bentov

Canada

Alexander Beristain

Canada

Agnieszka Blitek

Poland

\author{
Edson Borges \\ Brazil \\ Leon Brokken \\ Sweden \\ Theodore Brown \\ Canada
}

Richard Burney

United States of America

Alison Campbell

United Kingdom

Bruce Campbell

United Kingdom

Koel Chaudhury

India

Jerome Check

United States of America

Tereza Cindrova-Davies

United Kingdom

David Clark

Canada

Jacques Cohen

United States of America

Laurel Coons

United States of America

Robert Cushman

United States of America

\author{
Ivana Beatrice Da Cruz \\ Brazil
}

Petros Damos

Greece

Jan Danser

Netherlands

Tirtha Datta

India

Maurizio Dattilo

Switzerland

Frank De Jong

Netherlands

M L N Deepika

India

Gregory Dissen

United States of America

Marie-Madeleine Dolmans

Belgium

Erling Ekerhovd

Sweden

Gloria Evans

New Zealand

Kathleen Eyster

United States of America

Human Fatemi

Belgium

Correspondence: a_buko@comcast.net

The Institute of Biotechnology, Academy of Sciences of the Czech Republic,

Prague, Czech Republic 
Jens Fedder

Denmark

Stephen Ford

United States of America

Niamh Forde

Ireland

Jason Franasiak

United States of America

Anita Franczak

Poland

Leyland Fraser

Poland

David R. Garris

United States of America

Audrey Gaskins

United States of America

Francisco Gaytan

Spain

Ariane Germeyer

Germany

Vince Kornél Grolmusz

Hungary

Malgorzata Grzesiak

Poland

Roberto Gualtieri

Italy

Leah Hawkins

United States of America

Paul L Hermonat

United States of America

Linda Hong

United States of America

Ariel Hourvitz

Israel

Monika Kaczmarek

Poland

Fusako Kagitani

Japan

Hideo Kamomae

Japan

Frederick Kan

Canada

Suresh Kattera

Singapore
Jong-Min Kim

Korea, South

Kirstine Kirkegaard

Denmark

Kotaro Kitaya

Japan

Peter Kovacs

Hungary

Sharon Ladyman

New Zealand

Susan Laird

United Kingdom

Wei Lei

United States of America

Sheena Lewis

United Kingdom

Harry Lieman

United States of America

Yung-Ming Lin

Taiwan

Ewa Luszczek-Trojnar

Poland

Walid Maalouf

United Kingdom

Guido Macchiarelli

Italy

Bryce Maciver

United States of America

Matthew Mears

United Kingdom

Sergey Medvedev

United States of America

Roelof Menkveld

South Africa

Velja Mijatovic

Netherlands

Dean Morbeck

United States of America

Neela Mukhopadhaya

United Kingdom

Vanessa Murphy

Australia

Carolina Nastri

Brazil
Krzysztof Nowosielski

Poland

Cleida Oliveira

Brazil

Johannes Ott

Austria

Alessio Paffoni

Italy

Rosaura Pérez-Pé

Spain

Caitlyn Placek

United States of America

Josep V. Planas

Spain

Dwi Pujianto

Indonesia

Alberto Revelli

Italy

Anna Rising

Sweden

Fernanda Rocha

Canada

Geetanjali Sachdeva

India

Marie Saint-Dizier

France

Taiju Saito

Czech Republic

Smita Salian-Mehta

United States of America

Eleonora Salvolini

Italy

Daniele Santi

Italy

Regiane Santos

Netherlands

Heide Schatten

United States of America

Dietmar Schlembach

Germany

Richard Scott

United States of America

Balasubramanian Senthilkumaran India 
Ruijin Shao
Sweden

Gemma Sharp

United Kingdom

Marta Siemieniuch

Poland

Petra Sipilä

Finland

Mariusz Skoczynski

Poland

Berta Soldevila

Spain

Tamas Somfai

Japan

Astrid Stecher

Austria

Xoana Taboada

Spain
Toshifumi Takahashi

Japan

Alejandro Tapia-Pizarro

Chile

Juan J Tarin

Spain

Tyl Taylor

United States of America

Antoine Torre

France

Nam Tran

United States of America

Andreas Vernunft

Germany

Angela Vinturache

Canada

Angel Wang

China
Xin Wang

United States of America

Camilla Whittington

Australia

Izabela Woclawek-Potocka

Poland

Chadi Yazbeck

France

Marc Yeste

United Kingdom

Haiyang Yu

United States of America

Ying Zhang

China 